\title{
Optical Modeling of Black Silicon for Solar Cells Using Effective Index Techniques
}

\author{
Tasmiat Rahman ${ }^{(1)}$ and Stuart A. Boden
}

\begin{abstract}
Texturing the surface with both micro and nano scale features to form black silicon is a promising approach in improving solar cell efficiency. In optical modeling of such a surface, it is difficult to balance the accuracy and computational resource. In this work, we develop on a semianalytical model, effective index technique (EIT), which utilizes a finite-difference time domain (FDTD) method to represent the nanoscale texturing as an effective medium, and then apply this to microscale structures, which can then be modeled using the transfer matrix method and ray-tracing. We fabricate and model both periodic and random nanoscale textures, and analyze the accuracy of several effective index models against measured reflectivity. The limitations in the model are identified and coherency of the films is studied. The semianalytical method is shown to perform better than the other effective medium approaches for modeling black silicon and is applicable to modeling multiscale textures, whereas full numerical methods such as FDTD are not. However, although the EIT approach predicts the trends in antireflective performance of a texture, it remains inaccurate when compared with the experiment. Also, as with all effective medium approaches, the EIT does not account for light trapping through scattering.
\end{abstract}

Index Terms-Black Si, effective medium, finite-difference time domain (FDTD), nanoscale, optics, ray-tracing (RT), transfer matrix method (TMM).

\section{INTRODUCTION}

O PTICAL enhancement is a key factor in achieving efficient performance in solar cells, in particular for materials that suffer from high reflectivity such as silicon (Si). Several approaches are used to tackle this problem including antireflective coatings (ARCs) and microscale pyramidal texturing. Another approach to antireflection is to form arrays of nanoscale features on the surface. This reduces reflectivity to such an extent that these surfaces have been collectively termed black silicon [1]-[6]. These surfaces can be both periodic (e.g., well-defined pillars) or heterogeneous (e.g., grass-like), and formed on planar or pyramidal textures, reducing reflectivity over a broad spectral range and enhancing light trapping [7]-[10]. In order to optimize

Manuscript received July 3, 2017; revised August 15, 2017; accepted August 28, 2017. Date of publication September 26, 2017; date of current version October 19, 2017. The work of T. Rahman and S. A. Boden was supported by the Supersolar Solar Energy Hub under EPSRC Grant EP/J017361/1 and Grant EP/M014797/1. (Corresponding author: Tasmiat Rahman.)

The authors are with the Department of Electronics and Computer Science, University of Southampton, Southampton SO17 1BJ, U.K. (e-mail: t.rahman@soton.ac.uk; sb1@ecs.soton.ac.uk).

This paper has supplementary downloadable material available at http://ieeexplore.ieee.org

Color versions of one or more of the figures in this paper are available online at http://ieeexplore.ieee.org.

Digital Object Identifier 10.1109/JPHOTOV.2017.2748900 the aforementioned antireflective surfaces, [ARCs, microscale pyramids, nanowires (NWs)], several modeling techniques are available. ARCs can be efficiently modeled using the transfer matrix method (TMM) [11]. This uses a matrix to describe continuity conditions for the electric field across boundaries within a stack of layers (e.g., dielectrics on $\mathrm{Si}$ ). This significantly reduces the computation time in comparison to calculating the partial transmission and reflection through each of the various layers. This approach however does not include optical path enhancement through changes in propagation direction of light by textured surfaces. This can be modeled using ray-tracing (RT) which calculates the path of waves through discrete points, considering changes of direction through reflection and refraction at interfaces. This method is limited to structures with features greater than the incident wavelength. To compute nanoscale optics for solar cell applications where a broadband response is necessary, an efficient technique is to use the finite-difference time domain (FDTD) method. This discretizes the nanoscale structure into a Yee cell [12] and solves for the electromagnetic fields as a function of time before applying Fourier transforms to calculate the spectral response. Challenges arise when modeling multiscale surfaces such as nanoscale textures on microscale pyramidal surfaces. Modeling microscale textures using the FDTD technique demands considerable computational resource. Furthermore, for such large simulation domains, it is difficult to achieve convergence. On the other hand, RT can accurately model microscale features but is not valid for nanoscale features with dimensions below the ray optic limit. In this work, this problem is studied by treating the nanoscale surface as a single film using various effective medium techniques and then applying this to the microscale pyramidal surfaces. This multiscale hybrid structure can then be modeled using a combination of RT and TMM with tools such as Sentaurus TCAD [13]. Well-established effective medium models for representing the nanoscale textures, namely the volume averaging technique (VAT) [14], Maxwell-Garnett (MG) [15], and Bruggeman (BG) [16] are analyzed and compared with FDTD, along with a recently developed approach called the effective index technique (EIT) [17].

The models are first analyzed for various nanoscale feature radii, and their limitations identified. The use of EIT in representing periodic nanopillars is then explored, with modeled data compared with measured data of samples fabricated using nanoimprint lithography. The EIT model for pseudorandom NWs is then explored in an attempt to account for the heterogeneity found in many experimentally-realized nanoscale 


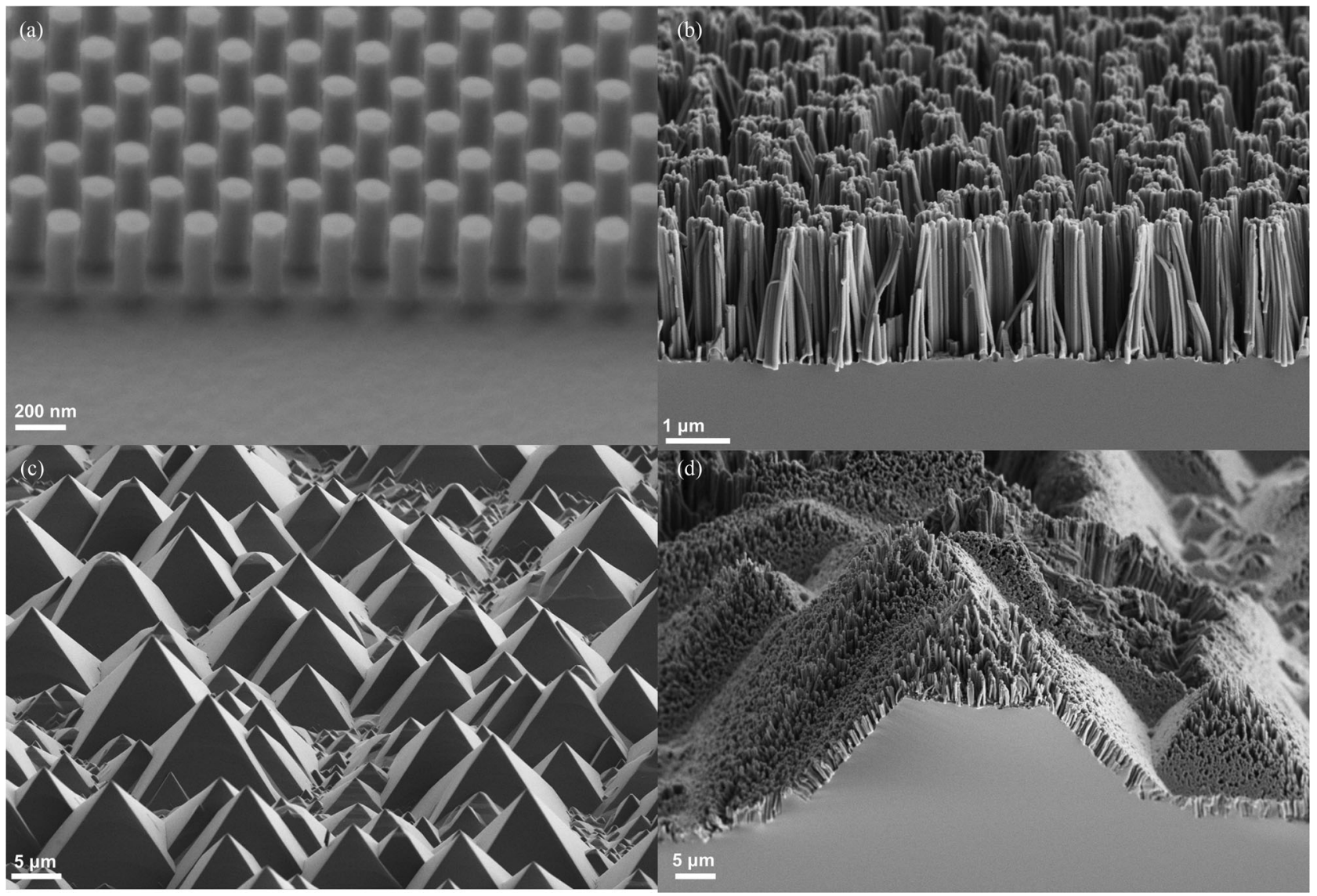

Fig. 1. Scanning electron micrographs of (a) periodic nanopillars fabricated via nanoimprint lithography, (b) random NWs fabricated via MACE, (c) pyramids fabricated via $\mathrm{KOH}$ etching, (d) multiscale hybrid structure of pyramids and random NWs fabricated via MACE+KOH etching.

textures being developed for silicon solar cell technologies. Furthermore, the influence of treating such films incoherently is studied. Finally, the effective medium films are then applied to pyramidal surfaces in Sentaurus TCAD and the results are compared with measured data over a wide angular range, from fabricated multiscale-textured surfaces. It should be clarified that whilst light trapping via scattering of incoming light by textured features is an important aspect of solar cell performance, none of the effective medium approaches (even EIT) takes this into account, and this work is limited to antireflection properties only.

\section{FABRICATION}

To verify the accuracy of the modeling techniques, both periodic and random nanoscale textured surfaces were fabricated and characterized. The periodic texture [see Fig. 1(a)] consisted of a hexagonal array of cylindrical pillars fabricated on a planar silicon surface with a pitch of $280 \mathrm{~nm}$, over an area of $1 \mathrm{~cm}^{2}$ using nanoimprint lithography and dry etching, as described in [18]. The random nanoscale-textured surface [see Fig. 1(b)] was fabricated by metal-assisted chemical etching (MACE), which results in dense arrays of randomly distributed but vertically aligned NWs. A pyramidal-textured surface [see Fig. 1(c)] was fabricated using standard alkaline etching. Finally, a multiscale hybrid sample [see Fig. 1(d)] was fabricated by first forming pyramids and then using MACE to create the nanoscale texture on the pyramidal surface.

The MACE process [1] occurs in two stages: the first is the nucleation stage in which $\mathrm{SiO}_{2}$ pits are formed underneath metal nanoparticles (in this work, Ag) deposited on the silicon surface. The second stage is to apply an etchant (in this work, $\mathrm{HF}$ ) to etch away the $\mathrm{SiO}_{2}$ and drive the nanoparticles deeper into the Si substrate. As this process proceeds, vertical NWs are formed. The etch rate can be regulated by temperature, which enables good control over NW length. The process begins with a cleaning step of a $5 \mathrm{~cm} \times 5 \mathrm{~cm}$ square Si sample $(\mathrm{Cz}$, 2.5-5 $\Omega-\mathrm{cm},<100>$ ) using Piranha etch solution of $\mathrm{H}_{2} \mathrm{SO}_{4}: \mathrm{H}_{2} \mathrm{O}$ in a 3:1 ratio followed by an HF dip. The sample is then placed in a silver nitrate $\left(\mathrm{AgNO}_{3}, 0.06 \mathrm{M}\right)$ and $\mathrm{HF}$ $(3 \mathrm{M})$ solution in a ratio of $1: 1$. An etch time of $2.5 \mathrm{~min}$ is used at a solution temperature of $50{ }^{\circ} \mathrm{C}$. The sample is then thoroughly rinsed in DI water followed by $5 \mathrm{~min}$ in nitric acid to remove Ag nanoparticles. The pyramidal-textured samples are fabricated by placing Piranha-cleaned wafers in a solution of $\mathrm{KOH}(0.2 \mathrm{M})$ and isopropanol at $70{ }^{\circ} \mathrm{C}$ for $60 \mathrm{~min}$. Alkaline etchants such as $\mathrm{KOH}$ exhibit a significantly faster etch rate on the $<100>$ than the $<111>$ planes of silicon, resulting in well-defined, randomly distributed pyramids on the surface of a $<100>$ wafer [19]. For fabricating the multiscale pyramid-NW samples, the pyramidal-textured samples are then subjected to the MACE process. Fig. 1 shows scanning 


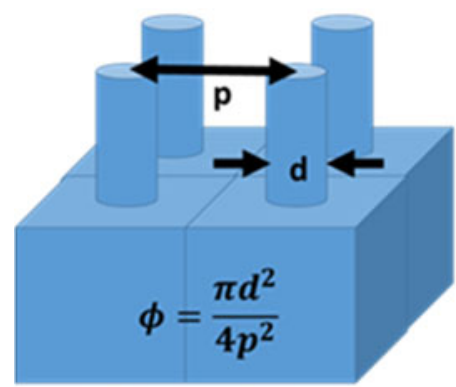

(a)

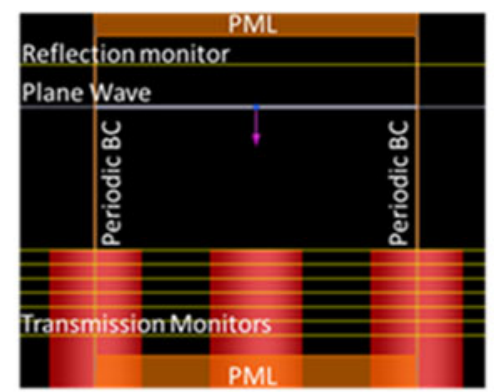

(b)

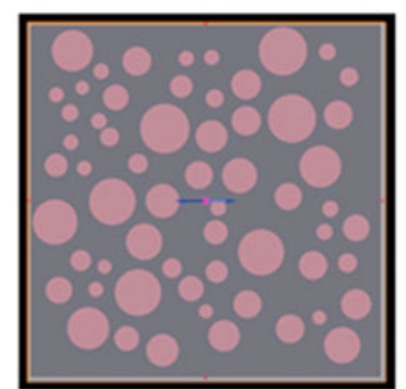

(c)

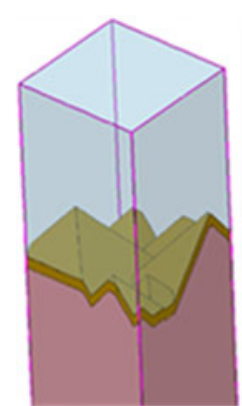

(d)

Fig. 2. (a) Schematic of unit cell and definition of fill ratio in effective medium models. (b) Schematic of semi-infinite NW in FDTD simulation domain. (c) Top down view of unit cell with NWs of various radii. (d) Schematic of pyramidal texture with deposited film of custom refractive index data for modeling in Sentaurus TCAD.

electron micrographs (SEM) of the fabricated samples: periodic nanopillars on planar silicon, random NWs on planar silicon, pyramids alone and the multiscale, hybrid texture with random NWs etched into pyramids. Measurements from the SEM images of the periodic arrays give a feature diameter of $140 \mathrm{~nm}$, a height of $360 \mathrm{~nm}$, and a pitch of $280 \mathrm{~nm}$. The heterogeneity of both the pyramidal and NW surface can be observed, whilst the periodic nanopillars are arranged in a hexagonal lattice.

Hemispherical reflectance measurements were conducted on each sample and then the samples were cleaved to enable morphological characterization by SEM (NVision40, Carl Zeiss). Hemispherical reflectance measurements were undertaken at an $8^{\circ}$ incident angle using an integrating sphere in a Bentham PVE300 photovoltaics characterization system, referenced to a Spectralon reflectance standard. An integrating sphere was also used in an custom-built system to measure reflectivity as a function of angle. A tungsten-halogen HL-2000-FHSA (Ocean Optics) lamp is used as light source, the samples were mounted in the center of the sphere on a rotatable clip and light was coupled out of the sphere via an optical fiber to a USB2000+ VIS-NIR-ES spectrometer (Ocean Optics). A bare, polished silicon sample was used as a reference.

\section{Modeling TechniQues}

To model the black silicon morphology as shown in Fig. 1, the first step is to begin with a periodic model and then to expand to include a degree of heterogeneity. Assuming a periodic structure, the nanopillar/NW can be modeled as a cylinder of a given diameter and height based on a cuboidal substrate. The structure can be simulated accurately using FDTD. We will explore whether faster simulation methods that involve defining the NW/air as a film on top of a silicon substrate, and then applying TMM and RT, can lead to accurate results. The FDTD tool used in this work to solve the spectral response of the NW surfaces is FDTD Solutions by Lumerical [20]. The NW is defined as a cylinder on a cuboidal substrate [see Fig. 2(a)]. Periodic boundaries are used in the $x$ - and $y$-directions to define the structure as an array of NWs, the pitch of which is determined by the width of the simulation domain. Perfectly matched layers (PML) are applied in the $z$-direction to define a semi-infinite substrate [see Fig. 2(b)]. The minimum mesh size of the Yee cell and the termination criteria of the simulation are set to $10 \mathrm{~nm}$ and $1 \mathrm{e}-5$, respectively. The heterogeneity was modeled using a unit cell of uniformly distributed random cylinders of varied radii $(20-80 \mathrm{~nm})$, position and height $(1 \mu \mathrm{m}$ $\pm 15 \mathrm{~nm}$ ), see Fig. 2(c). Effective medium approximations treat the NW surface as a thin film for which the effective refractive index $\left(n_{\text {eff }}\right)$ and the effective extinction coefficient $\left(k_{\text {eff }}\right)$ are the weighted averages of those for $\mathrm{Si}\left(n_{\mathrm{Si}}, k_{\mathrm{Si}}\right)$ and air $\left(n_{\mathrm{air}}, k_{\mathrm{air}}\right)$ based on the fill factor, $\phi$ (i.e., ratio of Si/air). The equations below represent the approximation for three different methods.

Volume Average Theory [14]:

$$
\begin{aligned}
A & =\phi\left(n_{\mathrm{si}}^{2}-k_{\mathrm{si}}^{2}\right)+(1-\phi)\left(n_{\mathrm{air}}^{2}-k_{\mathrm{air}}^{2}\right) \\
B & =2 n_{\mathrm{si}} k_{\mathrm{si}}+2 n_{\mathrm{air}} k_{\mathrm{air}}(1-\phi) \\
n_{\mathrm{eff}}^{2} & =\frac{1}{2}\left[A+\sqrt{A^{2}+B^{2}}\right] \\
k_{\mathrm{eff}}^{2} & =\frac{1}{2}\left[-A+\sqrt{A^{2}+B^{2}}\right] .
\end{aligned}
$$

Maxwell Garnett [15]:

$$
\epsilon_{\mathrm{eff}}^{2}=\epsilon_{\mathrm{si}}^{2}\left[1-\frac{3 \phi\left(\epsilon_{\mathrm{si}}^{2}-\epsilon_{\mathrm{air}}^{2}\right)}{2 \epsilon_{\mathrm{si}}^{2}+\epsilon_{\mathrm{air}}^{2}+\phi\left(\epsilon_{\mathrm{si}}^{2}-\epsilon_{\mathrm{air}}^{2}\right)}\right] .
$$

Bruggemann [16]:

$$
(1-\phi) \frac{\epsilon_{\mathrm{si}}^{2}-\epsilon_{\mathrm{eff}}^{2}}{\epsilon_{\mathrm{si}}^{2}-2 \epsilon_{\mathrm{eff}}^{2}}+\phi \frac{\epsilon_{\mathrm{air}}^{2}-\epsilon_{\mathrm{eff}}^{2}}{\epsilon_{\mathrm{air}}^{2}-2 \epsilon_{\mathrm{eff}}^{2}}=0 .
$$

Note that $\epsilon_{x}=\left(n_{x}+i k_{x}\right)^{2}$, where $x=$ air, Si, or eff.

Alongside these approximations, the EIT also approximates refractive index data for NWs. This semianalytical model presented in [17] calculates refractive index data by first modeling the NW as semi-infinite (i.e., no substrate but instead the base of the NW extending through the PML layer) and obtaining its reflected power $R$ and transmitted power $T$, through various depths $Z$ of the NW using the FDTD tool. An increase in the number of PML at the base of the simulation domain is necessary in this case to remove instabilities that are triggered at the interface between the dispersive silicon NWs and the PML boundary. The absorption coefficient $\alpha$ of the NW array can be related to the transmitted power at varying depths using the cutback technique described in [17], based on the Beer-Lambert 


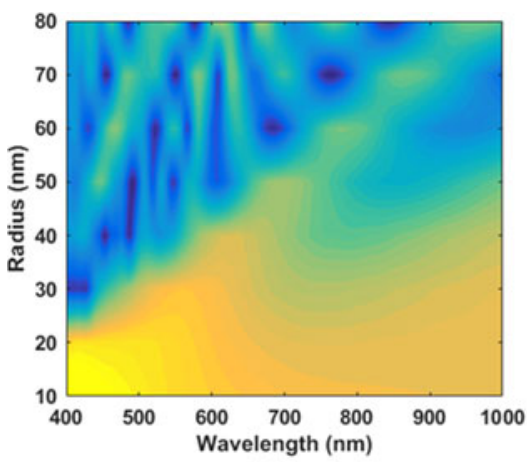

(a)

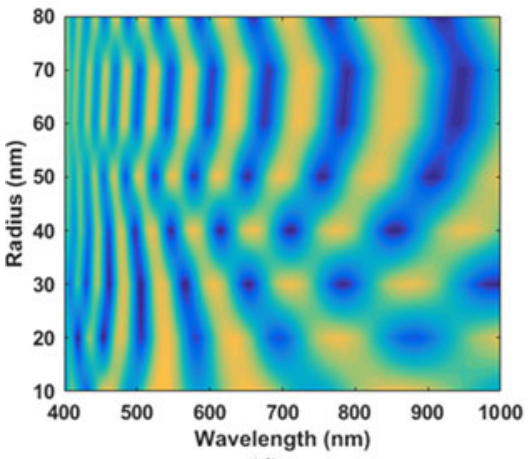

(d)

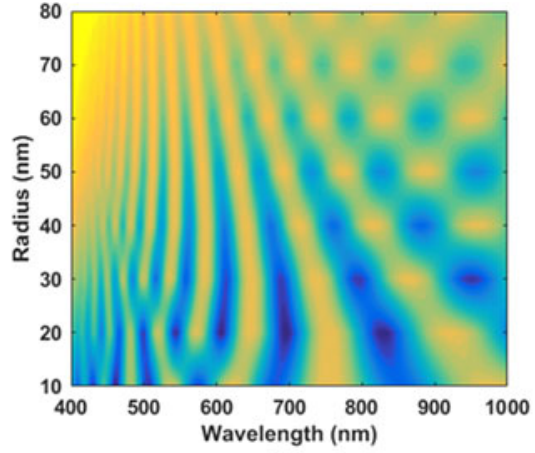

(b)

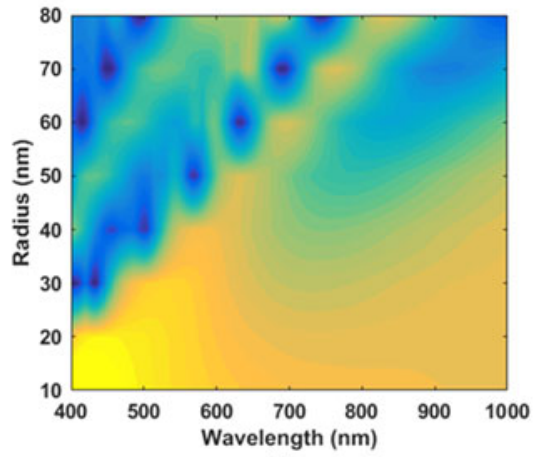

(e)

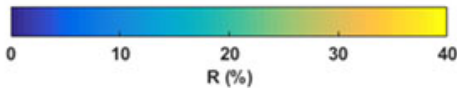

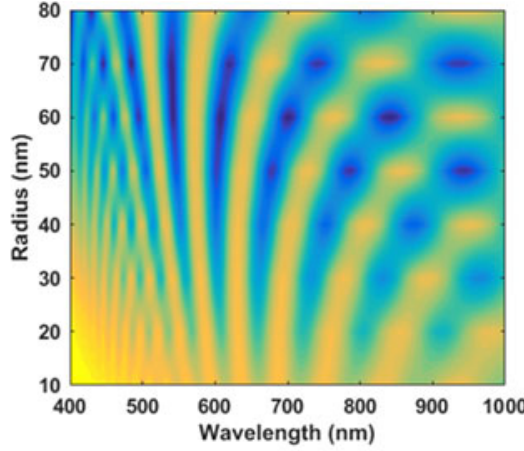

(c)

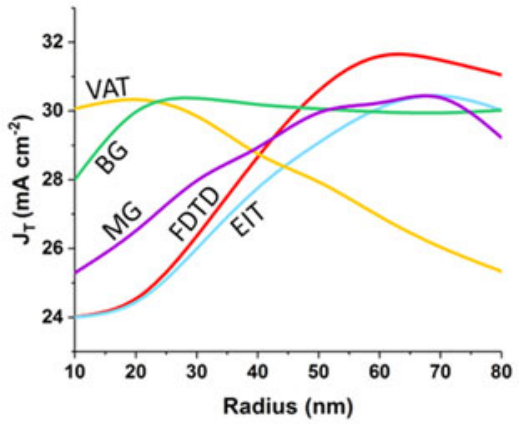

(f)

Fig. 3. Calculated reflectivity spectra for the periodic nanopillar structure with various pillar radii using (a) FDTD model, (b) VAT model, (c) BG model, (d) MG model, (e) EIT model, (f) $J_{T}$ as a function of various radii using the different models.

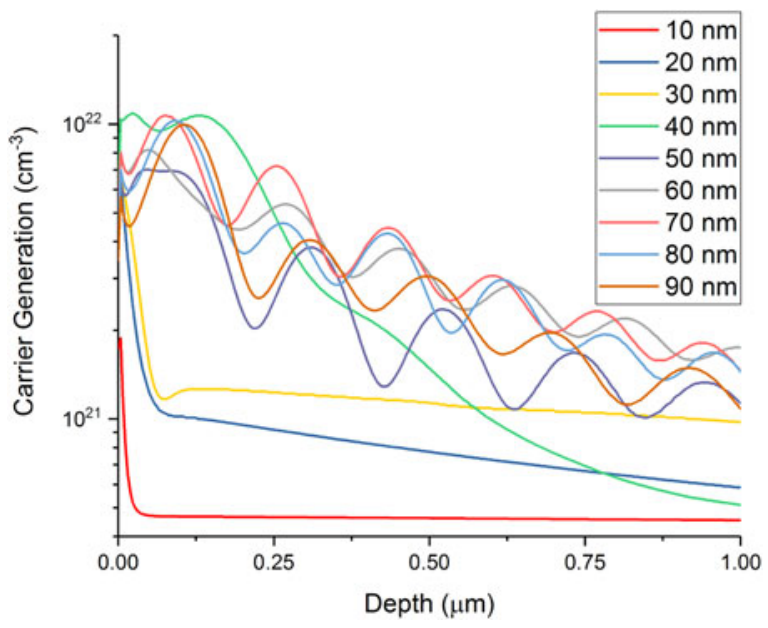

Fig. 4. Simulated carrier generation (using FDTD) as a function of depth within a nanowire of various radii.

law, giving the following:

$$
\alpha=\frac{d(\ln T)}{d Z} .
$$

The extinction coefficient $k$ can then be calculated by

$$
k=\frac{\alpha \lambda}{4 \pi}
$$

Finally, the refractive index $n$ can be calculated by relating $k$ to the reflected power:

$$
R=\frac{(n-1)^{2}+k^{2}}{(n+1)^{2}+k^{2}} .
$$

The RT tool used in this work is Sentaurus TCAD device solver. Microscale texturing is defined as a random array of pyramids with base widths ranging from 1 to $5 \mu \mathrm{m}$ [see Fig. 2(d)]. The boundary conditions used are fully reflective in the $x-y$ plane and fully absorbing in the $z$ plane. The nanoscale textures are defined as a film deposited on top of the pyramids. These films have a custom-defined refractive index that has been precalculated using the aforementioned effective medium models. Furthermore, this tool can treat the films either coherently or incoherently.

\section{RESULTS}

Fig. 3(a) shows the reflectivity spectra obtained using the full FDTD technique (Lumerical) to solve differing radii of an NW unit cell (height $=500 \mathrm{~nm}$, period $=200 \mathrm{~nm}$ ). Likewise, Fig. 3(b)-(d) illustrates the reflectivity spectra of differing NW radii when using the VAT, MG, and BG models, respectively, to represent the NW unit cells as an effective medium before solving, using the TMM technique. The results of using the EIT technique to calculate the reflectivity spectrum as a function of varying radii are presented in Fig. 3(e). The results from the conventional effective medium models (i.e., VAT, MG, and BG) exhibit fringes which stem from the Fabry-Perot resonances 
due to interference of light reflected from the top and bottom of the NW layer [21]. However, it is observable that these fringes differ substantially from the FDTD result. In contrast, the EIT technique shows similarity to FDTD, illustrating guided modes that become red-shifted with increasing radii. This is because the EIT technique takes into account, not only geometry as is the case for the effective medium models, but also the influence of the electromagnetic fields of the neighboring NWs. As a means to characterize the similarity of the effective models and techniques to that of FDTD, the photon current from transmitted light into device $\left(J_{T}\right)$ is calculated based on the simulated reflectivity $(R)$ using the following relationship:

$$
J_{T}=-q \int_{\lambda_{\min }}^{\lambda_{\max }} \Phi(\lambda)(1-R(\lambda)) \operatorname{IQE}(\lambda) d \lambda
$$

whereby $\Phi$ is the incident photon flux density for AM1.5 spectrum, IQE is the internal quantum efficiency (assumed 1), and $q$ is the charge of an electron. The $J_{T}$ as a function of NW radii for the different modeling approaches is plotted in Fig. 3(f). This shows that of the four effective modeling approaches, the EIT model exhibits the best agreement with FDTD for smaller radii. However, this similarity worsens with increasing radius, and approaches the values of the BG and MG models for radii above $60 \mathrm{~nm}$. The VAT model remains significantly different to FDTD results across all radii, whilst MG and BG improve for larger radii but still exhibit errors $>1 \mathrm{mAcm}^{-2}$ for $J_{T}$.

The divergence in reflectivity for larger radii in EIT compared with FDTD can be accounted for by the complexity in carrier generation profile, as observable in Fig. 4. This shows onedimensional carrier generation profile as a function of depth (along $z$ ) into the NW, measured at the center point (of the $x-y$ plane). The profiles for radii $<30 \mathrm{~nm}$ show a sharp drop followed by a constant decay. This can be accounted for due to small radii only supporting fundamental modes and hence producing a relatively simple depth profile [5]. When using the cut-back technique with transmission monitors along the $z$-axis, a fit can be made using an absorption coefficient which is representative of this decay. As the radius increases, higher order modes emerge (see supplementary information), which yield carrier generation profiles that diverge away from a simple decay and thus lead to errors when fitting using the cut-back technique. Despite the differences ( $<3 \%$ error in $J_{T}$ ) for larger radii, the trend is similar across all radii when looking at both reflectivity and $J_{T}$. This information is still retained in the cut-back technique as this is averaging the transmitted power across the $x-y$ plane, and thus an overall decay representative of the NW is observed for which an absorption coefficient can be fitted.

Fig. 5 shows the measured reflectivity, as well as modeled data using FDTD and EIT of the periodic nanopillars fabricated via nanoimprint lithography. The morphology of this structure, which is shown in Fig. 1(a), is cylindrical with an average radius of $70 \mathrm{~nm}$ and height of $360 \mathrm{~nm}$. The array is a hexagonal lattice with a pitch of $280 \mathrm{~nm}$. The reflectivity measurement shows reasonable agreement with the FDTD modeled result, with similarities observable with the EIT modeled data (particularly the presence of peaks and troughs for $600 \mathrm{~nm}<\lambda<800 \mathrm{~nm}$ ). As the radius of the NW is $>30 \mathrm{~nm}$, the results using EIT model

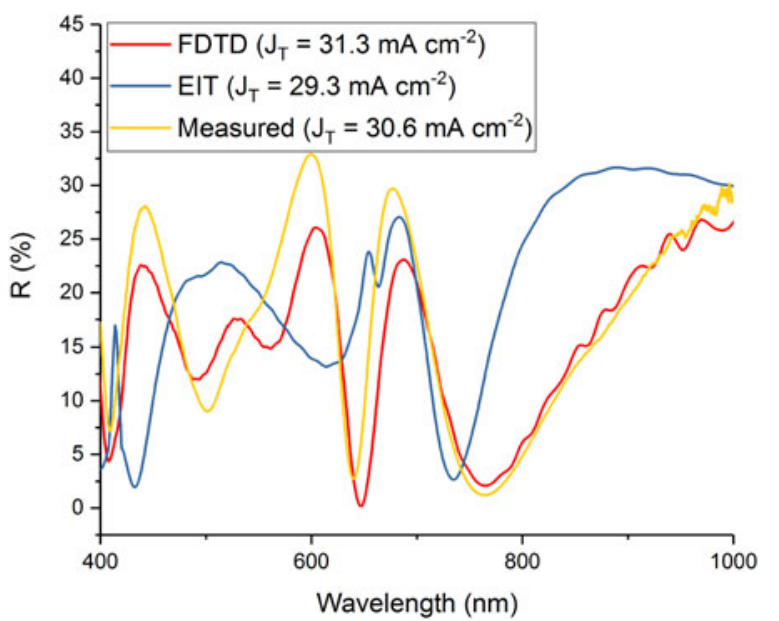

Fig. 5. Reflection spectra (modeled and measured) for a periodic nanowire array $($ pitch $=280 \mathrm{~nm}$, height $=360 \mathrm{~nm}$, radius $=70 \mathrm{~nm}$ ).

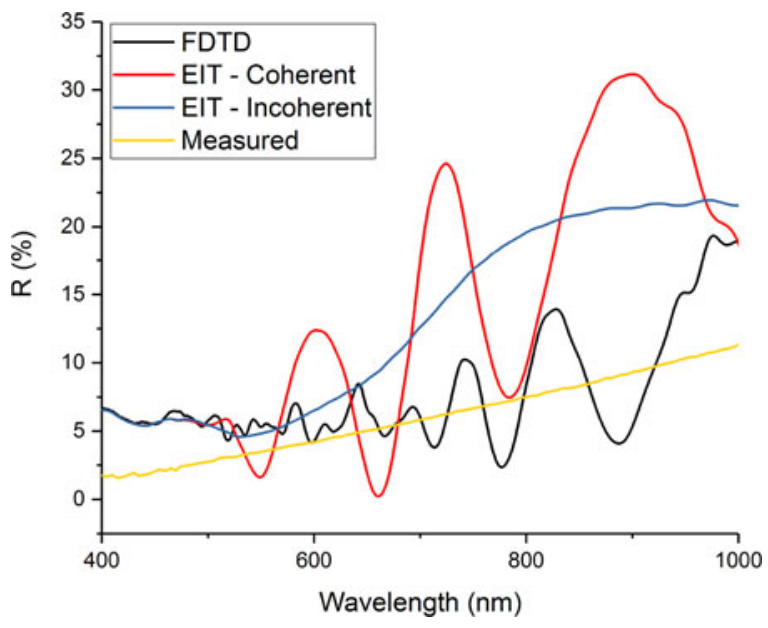

Fig. 6. Reflection spectra for random nanowires (average height $=1 \mu \mathrm{m}$ ) fabricated using MACE (measured data) or modeled as a pseudorandom array using FDTD and EIT approaches.

for this case are prone to the aforementioned limitations. This results in an error $<5 \%$ in the $J_{T}$ values between measured data and EIT modeling results.

Fig. 6 shows the measured reflectivity, as well as modeled data, using FDTD and EIT of the black silicon surface fabricated via MACE. The morphology of this structure, which is shown in Fig. 1(b), is heterogeneous with NWs varying in diameter from 30 to $200 \mathrm{~nm}$ and having heights of approximately $1 \mu \mathrm{m}$. The measured data show significantly reduced reflectivity compared with the periodic structure, which is the result of superposition of modes triggered by the various radii in this heterogeneous surface. The reduction in reflectivity is also observed in the modeled FDTD data, however it does not exhibit the broadband flat response observed experimentally. This is due to the limitation of pseudorandomness in a unit cell that can be efficiently modeled. The EIT modeled data have good agreement with FDTD for only small wavelengths. The error observed in this can be attributed to the limitations observed in the periodic case for larger diameters. The superposition of 


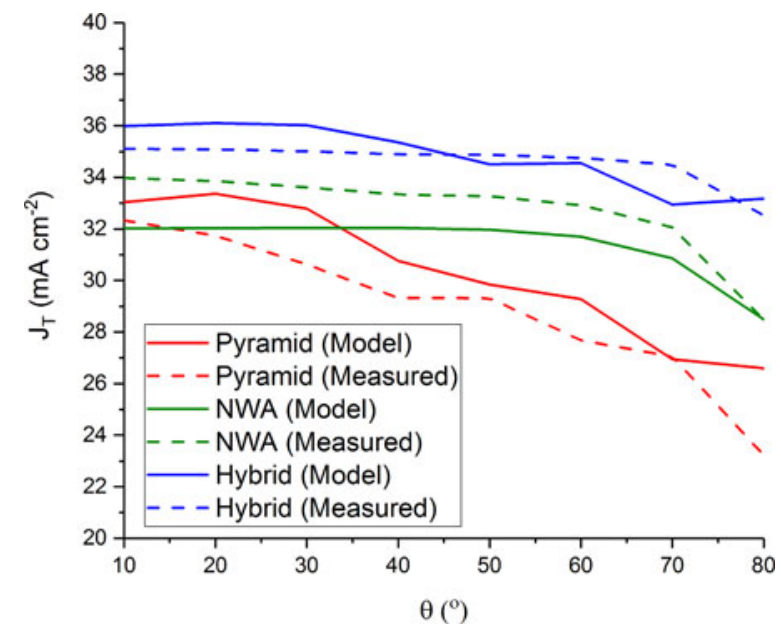

Fig. 7. Calculated $J_{T}$ at various angles of incidence for the different textured surfaces. This is based on spectral reflectivity modeled using Sentaurus TCAD with EIT films to define the nanoscale structures and fabricated samples measured in an integrating sphere. (See Supplementary material for reflectance spectra from which these results were derived).

different modes triggered by the various radii, produces carrier generation profiles that will diverge from a simple decay capable of being fitted using the cut-back technique. Both the FDTD and EIT modeled data exhibit peaks and troughs that are not present in the experimental measurement. The heterogeneity and the height of the black Si surface can result in an incoherent behavior of the NW array on silicon. As such, specifying the film as incoherent in the TCAD simulation (EIT) produces a flatter response with trends analogous to the experimental data. However, the resulting spectrum still does not exhibit the low reflectivity observed in the experimental case. The benefit of using EIT becomes more apparent when modeling multiscale textured surfaces under varying angles of light injection $(\theta)$. This is due to the enhanced simulation complexity involved when modeling using the full FDTD technique which includes having to use fixed wavelengths (angle of incidence of the source changes as a function of frequency for nonzero injection angles [20]), Bloch boundary conditions to account for phase changes across the unit cell [20] and increased PMLs to counter reflections from steep angles [20]. These complexities make it even more difficult to model a microscale structure with a nanoscale mesh as the both RAM and CPU usage become intensive.

Fig. 7 shows $J_{T}$ of the various textured surfaces over a range of angles of light injection (normalized to planar silicon). This is based on spectral reflectance data which can be found in Supplementary information. The modeled EIT results show a drop in $J_{T}$ of $20 \%, 12 \%$, and $8 \%$ from $\theta=10^{\circ}$ to $\theta=80^{\circ}$ for pyramidal, NW and multiscale hybrid textures, respectively. In comparison, the experimental results showed a drop in $J_{T}$ of $26 \%, 16 \%$, and $7 \%$, respectively. The retention of low reflectivity over large incident angles is one of the key benefits reported in the literature for black silicon surfaces [6], and this model successfully predicts this trend. It should be noted that whilst the trends are visible in the modeled data, absolute values do not have good agreement with those from experiments. One key factor is the lack of heterogeneity in the model compared with that of the fabricated samples.

\section{CONCLUSION}

In this paper, we present the use of an EIT model which uses a cut-back approach with the FDTD method to calculate the refractive index of a semi-infinite NW array. This has produced more accurate results when treating the NWs as an effective medium compared with VAT, BG, and MG effective medium models. However, the accuracy of the EIT model remains limited to smaller diameters, where only the fundamental modes are triggered. Higher modes result in carrier generation profiles that cannot be easily fitted to an exponential decay and as such limit the accuracy of the model. Reflectivity for periodic nanopillars (70 $\mathrm{nm}$ radius) fabricated by nanoimprint lithography showed agreement with FDTD results, and similarity in peak/trough distribution with the EIT model, with errors $<5 \%$ in the $J_{T}$. Measured reflectivity of black silicon surfaces fabricated by MACE showed significantly lower and flatter broadband response than the periodic case. Whilst pseudorandom NWs modeled in FDTD exhibited reduced reflectivity compared with periodic structures, the lack of heterogeneity impeded a lower, flatter broadband response. Furthermore, the EIT model failed to show agreement with FDTD for large $\lambda$. Treating the films incoherently produced a flatter response with trends similar to experimental data. Multiscale texture modeling at various angles of incidence using the EIT model with RT, showed drops in $J_{T}$ of $20 \%, 12 \%$, and $8 \%$ from $\theta=10^{\circ}$ to $\theta=80^{\circ}$ for pyramidal, NW, and multiscale textures, respectively. This retention of low reflectivity over a large angle range was also apparent in the experimental data.

In conclusion, EIT performs better than the other effective medium approaches for modeling black silicon and is applicable to modeling multiscale textures, whereas FDTD alone is not. Whilst general trends in overall AR performance of a texture can be predicted with the EIT technique, it remains inaccurate when compared with experiment for determining absolute reflectivity, increasingly so as the nanoscale feature radius exceeds $30 \mathrm{~nm}$. Also, as with all EMT methods, the EIT technique does not account for light trapping through scattering.

\section{ACKNOWLEDGMENT}

The authors would like to thank NIL Technology Ltd. for fabricating the periodic nanopillar array presented here. The data from this paper can be obtained from the University of Southampton e-Prints repository (http://dx.doi.org/10.5258/ SOTON/D0187).

\section{REFERENCES}

[1] K. Q. Peng and S. T. Lee, "Silicon nanowires for photovoltaic solar energy conversion," Advanced Mater, vol. 23, no. 2, pp. 198-215, 2011.

[2] M.-L. Zhang, et al., "Preparation of large-area uniform silicon nanowires arrays through metal-assisted chemical etching," J. Phys. Chem. C, vol. 112, no. 12, pp. 4444-4450, 2008.

[3] H. Savin et al., "Black silicon solar cells with interdigitated back-contacts achieve $22.1 \%$ efficiency," Nature Nanotechnol., vol. 10, no. 7, pp. 624$628,2015$. 
[4] R. S. Davidsen et al., "Black silicon laser-doped selective emitter solar cell with $18.1 \%$ efficiency," Solar Energy Mater. Solar Cells, vol. 144, pp. 740-747, 2016.

[5] T. Rahman, M. Navarro-Ca, and K. Fobelets, "High density micropyramids with silicon nanowire array for photovoltaic applications," Nanotechnology, vol. 25, no. 48, 2014, Art. no. 485202.

[6] T. Rahman et al., "Passivation of all-angle black surfaces for silicon solar cells," Solar Energy Mater. Solar Cells, vol. 160, pp. 444-453, 2017.

[7] P. Spinelli, M. A. Verschuuren, and A. Polman, "Broadband omnidirectional antireflection coating based on subwavelength surface Mie resonators," Nature Commun., vol. 3, 2012, Art. no. 692.

[8] A. Ingenito, I. Olindo, and M. Zeman, "Nanocones on micropyramids: Modulated surface textures for maximal spectral response and high efficiency solar cells," Prog. Photovolt., Res. Appl., vol. 23, no. 11, pp. 16491659, 2015.

[9] M. Kroll et al., "Black silicon for solar cell applications," Proc. SPIE., vol. 8438,2012 , Art. no. 843817.

[10] A. Bett et al., "Wave optical simulation of the light trapping properties of black silicon surface textures," Opt. Express, vol. 24, no. 6, pp. A434 A445, 2016.

[11] C. C. Katsidis and D. I. Siapkas, "General transfer-matrix method for optical multilayer systems with coherent, partially coherent, and incoherent interference," Appl. Opt., vol. 41, no. 19, pp. 3978-3987, 2002.

[12] K. S. Yee, "Numerical solution of initial boundary value problems involving Maxwell's equations in isotropic media," IEEE Trans. Antennas Propag, vol. AP-14, no. 3, pp. 302-307, May 1966.

[13] Sentaurus TCAD Version K-2015.06, Synopsys Inc., Mountain View, CA, USA, 2015.
[14] J. de Ro and S. Whitaker, "Maxwells equations in two-phase systems I: Local electrodynamic equilibrium," Transp. Porous Media, vol. 39, no. 2, pp. 159-186, 2000.

[15] J. M. Garnett, "Colours in metal glasses, in metallic films, and in metallic solutions. II,” Phil. Trans. Roy. Soc. London Ser. A, vol. 205, pp. 237-288, 1906.

[16] V. D. Bruggeman, "Berechnung verschiedener physikalischer Konstanten von heterogenen Substanzen. I. Dielektrizittskonstanten und Leitfähigkeiten der Mischkörper aus isotropen Substanzen," Annalen der physik, vol. 416, no. 7, pp. 636-664, 1935

[17] S. Patchett et al., "Effective index approximation for ordered silicon nanowire arrays," J. Opt. Soc. Amer. B, vol. 30, no. 2, pp. 306-313, 2013

[18] S. A. Boden and D. M. Bagnall, "Nanostructured biomimetic moth-eye arrays in silicon by nanoimprint lithography," Proc. SPIE, vol. 7401, 2009, Art. no. $74010 \mathrm{~J}$.

[19] D. L. King and M. E. Buck, "Experimental optimization of an anisotropic etching process for random texturization of silicon solar cells," in Proc. Conf. Rec. 22nd IEEE Photovolt. Spec. Conf., 1991, pp. 303-308.

[20] F. Lumerical, Solutions. "Web source," 2012. [Online]. Available: https://www. lumerical. com/tcad-products/fdtd/

[21] H. Alaeian, A. C. Atre, and J. A. Dionne, "Optimized light absorption in Si wire array solar cells,” J. Opt., vol. 14, no. 2, 2012, Art. no. 024006.

Authors' photographs and biographies not available at the time of publication. 